\title{
Cortisol Levels and Gastrointestinal Disorders After Stressful Surgery in Rabbits
}

\author{
SEOK JIN JANG ${ }^{1 *}$, SEONG SOO KANG ${ }^{2 *}$, SOO JIN SON ${ }^{1}$, JAE YEON LEE ${ }^{1}$, \\ GONHYUNG KIM ${ }^{1}$ and SEOK HWA CHOI ${ }^{1}$ \\ ${ }^{1}$ Department of Veterinary Surgery, College of Veterinary Medicine, \\ Chungbuk National University, Cheongju, Republic of Korea; \\ ${ }^{2}$ Veterinary Teaching Hospital, College of Veterinary Medicine, \\ Chonnam National University, Gwangju, Republic of Korea
}

\begin{abstract}
Rabbits are widely used in biomedical studies because they are docile and manageable. However, they are prone to gastrointestinal disorders due to their vulnerability to stress. Eighteen adult rabbits were used for allogenic tracheal surgery. The tracheas for allograft, 20-mm length, were transplanted into 20-mm tracheal defects. Immediately after surgery, most rabbits suffered from poor appetite. Food and water intake gradually recovered within 7 days after surgery, but six rabbits had severe anorexia from day 7 postsurgery. Four of these rabbits developed symptoms of diarrhea after surgery; three of them died several days after the onset of diarrhea, while one rabbit recovered. Gastrointestinal disorders need to be prevented in rabbits undergoing stressful surgery. Furthermore, it is important to choose the proper type and dose of analgesics in order to relieve postoperative pain. With this observation in mind, rabbits are not considered to be an appropriate model for stressful surgery.
\end{abstract}

Rabbits are docile and easy to handle for biomedical studies, but very sensitive to stress $(1,2)$. Gastrointestinal (GI) disorders are potentially fatal conditions commonly seen in rabbits. When the GI tract slows-down or stops moving for some reason, the water of the gastric contents is extracted from the stomach and the gastric contents become dry,

This article is freely accessible online.

*These Authors contributed equally to this work.

Correspondence to: Seok Hwa Choi, DVM, Ph.D., Professor, Department of Veterinary Surgery, College of Veterinary Medicine, Chungbuk National University, Cheongju, 28644, Republic of Korea. Tel: +82 432613144, Fax: +82 432613224, e-mail: shchoi3144@hanmail.net

Key Words: Gastrointestinal disorder, surgery, stress, rabbits, cortisol. compact and thick. Therefore, the content mass is unable to pass normally through the GI tract, creating a blockage $(3,4)$. Harmful intestinal bacteria proliferate and build up gas in the GI tract, causing significant pain to the rabbit. The bacteria also produce toxins that can damage the liver. In addition, hepatic lipidosis can occur because the rabbit goes into negative energy absorption $(5,6)$. Rabbits with GI disorders show the clinical symptoms of anorexia, weight loss, diarrhea, depression, and abdominal distension $(2,5,6)$. If left untreated or improperly treated, a rabbit with a GI disorder may die with pain within $24 \mathrm{~h}$. The causes of GI disorders include an improper diet, pain, stress, dental problems, lack of exercise, and long-term use of antibiotics $(3,5,7,8)$. Among these, stress is particularly important in rabbits used for biomedical studies because they are very sensitive to stress. Inappropriate housing environment and diet, surgery, pain and treatment, such as injections for experiments, may be stressful to laboratory rabbits. This study describes the occurrence of GI disorders and cortisol levels after stressful surgery in rabbits.

\section{Materials and Methods}

Animals. Eighteen 18-week-old male New Zealand White rabbits (Samtaco Lab., Osan, Korea) weighing 2.5 - $2.7 \mathrm{~kg}$ were used for allogenic tracheal transplantation. All rabbits were acclimated for 2 weeks in the animal house at the Laboratory Animal Research Center of Chungbuk National University (CBNU), Korea. Water and food were supplied ad libitum during the experimental period. The rabbits were housed in individual cages, with 12-hour light-dark intervals. The room temperature and humidity were maintained at $20 \pm 2^{\circ} \mathrm{C}$ and $50 \pm 20 \%$, respectively. This experiment was approved by the Institutional Animal Care and Use Committee of CBNU (CBNU-822-15-01). No symptoms were observed in any rabbits during the 2-week acclimation period in the animal house at Laboratory Animal Research Center of CBNU.

Surgery and medication. All rabbits were administered 0.05 $\mathrm{mg} / \mathrm{kg}$ atropine (Atropine sulfate Inj.; Jeil Pharm Co., Seoul, Korea), 3 mg/kg tramadol (Maritrol Inj.; Jeil Pharm Co., Seoul, 

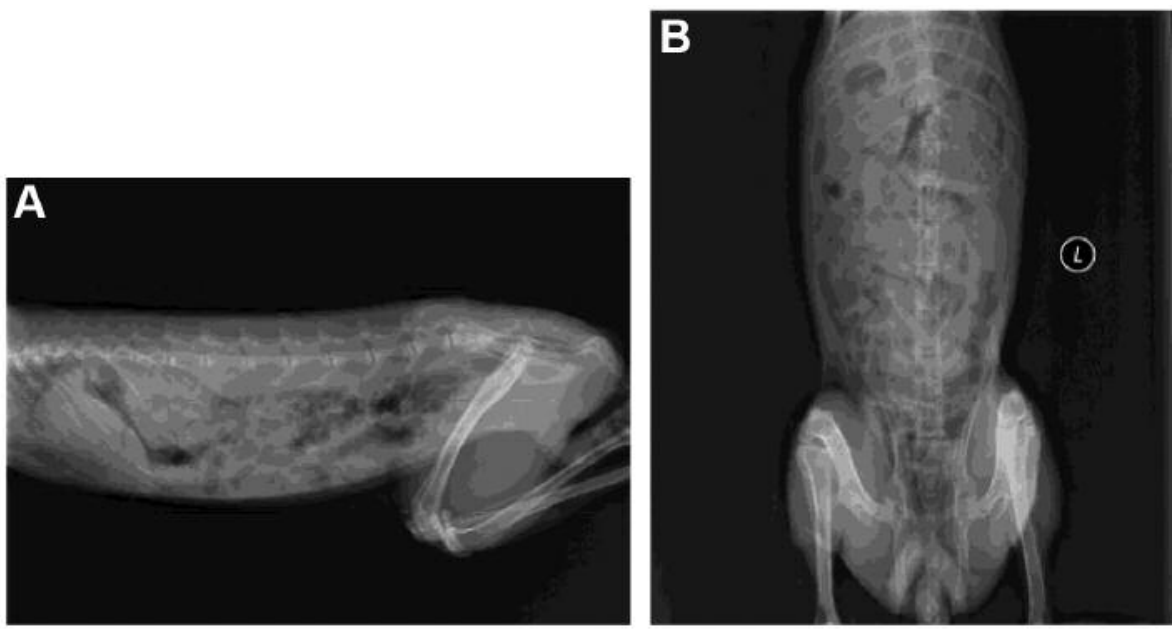

Figure 1. Abdominal radiographic images of the New Zealand White rabbits with gastrointestinal disorder. Moderate gastric dilation and intestinal gas are apparent in the right lateral $(A)$ and ventrodorsal $(B)$ views.
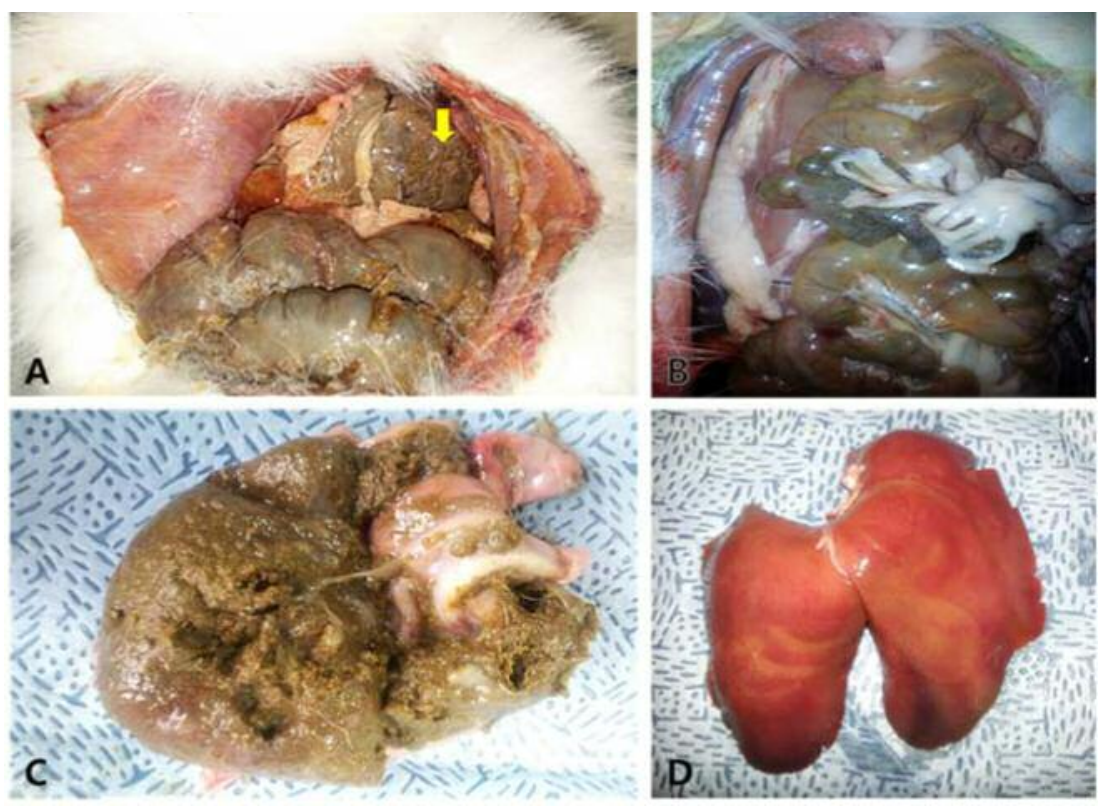

Figure 2. Autopsy findings of gastric dilation and perforation (A, arrow), intestinal track filled with gas (B), a mass of food and hair in the stomach $(C)$, and fatty yellow liver $(D)$ in New Zealand White rabbits with gastrointestinal disorder.

Korea), $0.01 \mathrm{mg} / \mathrm{kg}$ glycopyrrolate (Glycopyrrolate Inj.; Reyon Pharm Co., Seoul, Korea), and $10 \mathrm{mg} / \mathrm{kg}$ amoxicillin (Foxolin Inj.; Samjin Pharm Co., Seoul, Korea) intramuscularly for premedication 30 minutes before inducing anesthesia. Anesthesia was induced intramuscularly using a combination of $10 \mathrm{mg} / \mathrm{kg}$ tiletamine-zolazepam (Zoletil, Virbac, France) and $5 \mathrm{mg} / \mathrm{kg}$ xylazine (Rompun, Bayer Korea, Seoul, Korea). The tracheas for allograft, 20-mm length, was harvested from donor New Zealand White rabbits, transplanted into $20-\mathrm{mm}$ tracheal defects, and sutured with 5-0 polyglyconate. Postoperative medications, administered once a day for 3 days, were tramadol $(3 \mathrm{mg} / \mathrm{kg})$, glycopyrrolate $(0.01 \mathrm{mg} / \mathrm{kg})$, and amoxicillin $(10 \mathrm{mg} / \mathrm{kg})$. The animals were observed for 8 weeks after surgery.

Diagnoses of GI disorders. After surgery, some rabbits showed diarrhea, anorexia, weight loss, and depression. Rabbits with clinical symptoms of GI disorders were subjected to radiography, autopsy, and histopathology to confirm the diagnoses of GI disorders. The 


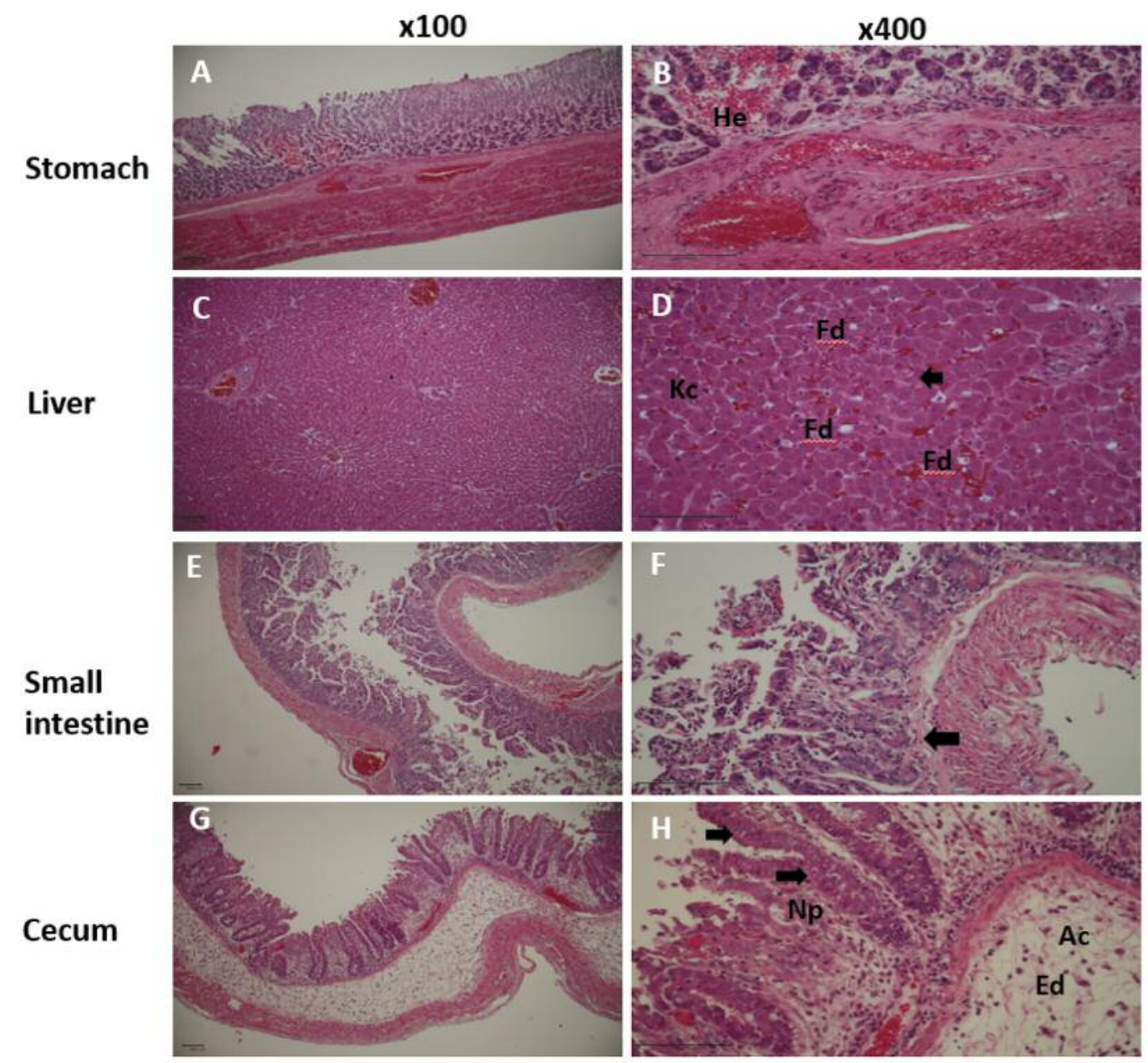

Figure 3. Histological analysis of the digestive system in New Zealand White rabbits with gastrointestinal disorder. Hemorrhagic gastric mucosa $(\mathrm{He})$ in the stomach was observed. Activated Kupffer cells $(K c)$, fat droplets $(F d)$ and apoptotic bodies (arrow) were observed in the liver. Small intestinal mucosa was edematous (arrow) and sloughed. Colonic mucosa was severely edematous (Ed) and sloughed with the presence of a number of neutrophils (Np), lymphocytes (arrows), and apoptotic cells (Ac). H\&E staining.

serum cholesterol level was measured in all rabbits to identify stress. Radiography was performed to identify stagnant gas in the intestine and lumps of food in the stomach. The rabbits were given midazolam (Midazolam inj., Bukwang Pharm Co., Seoul, Korea, $0.25 \mathrm{mg} / \mathrm{kg}$ ) intramuscularly for sedation prior to imaging. Abdominal radiography was obtained with X-ray (KODAK Directview CR500 system, Eastman Kodak Co., USA). Autopsies were performed as soon as affected rabbits died. Simultaneously, sampling of tissue specimens for histopathological examination was carried out. The stomach, liver, small intestine and cecum were sampled and then fixed in $10 \%$ neutral buffered formalin for hematoxylin and eosin (H\&E) staining.

Measurement of cortisol level. Cortisol levels were tested using Roche Modular Analytics E170 (Roche Diagnostics, Mannheim, Germany). Blood sampling for serum cortisol measurement was carried out during the same time periods on days 1 and 7 before and after surgery. Blood samples were collected from the auricular vein in rabbits.
Statistical analysis. Statistical analyses were performed using SPSS statistical software package version 19.0.1.1 (IBM SPSS Statistics for Window, Version 19.0; IBM Corp., Armonk, NY, USA). Data are presented as the mean \pm standard deviation (SD). Normality and homogeneity of the data were confirmed before analysis of variance (ANOVA). Differences among the experimental groups were assessed by one-way ANOVA followed by Duncan's multiple range tests. Null hypotheses of no difference were rejected if $p$-values were less than 0.05 .

\section{Results and Discussion}

The welfare of animals used for biomedical research is very important. There are good ethical, scientific, legal and economic reasons to ensure that animals are properly monitored and used in minimum numbers. Good science and good animal welfare are compatible. If the animal suffers from stress or pain, it may affect the results of biomedical study. 
Table I. Serum cortisol levels $(\mu \mathrm{g} / \mathrm{dl})$ of New Zealand White rabbits after surgery.

\begin{tabular}{lccc}
\hline Group & \multicolumn{3}{c}{ Days after surgery } \\
\cline { 2 - 4 } & 0 & 1 & 7 \\
\hline Normal $(\mathrm{n}=11)$ & $1.32 \pm 0.25$ & $2.66 \pm 0.31$ & $1.88 \pm 0.33$ \\
GI disorder $(\mathrm{n}=6) \S$ & $1.20 \pm 0.49$ & $2.81 \pm 0.87$ & $2.76 \pm 1.40^{*}$ \\
\hline
\end{tabular}

GI: Gastrointestinal. §One out of 7 rabbits died within 1 week and was excluded from the analysis. Data are the mean \pm S.D. * Significantly different from normal group at $p<0.05$.

In this study, almost all rabbits showed anorexia immediately after allogenic tracheal transplantation. Food and water intake gradually recovered within 7 days after surgery, but six out of the 18 rabbits developed severe anorexia from day 7 post-surgery. Rabbits who had diarrhea within 1 week after surgery were worse than rabbits who had diarrhea after 1 week. Four of these rabbits developed symptoms of diarrhea after surgery; three of them died several days after the onset of diarrhea, while one rabbit recovered. The diarrheal rabbits were radiographed and GI disorder was confirmed by intraperitoneal findings. In the right lateral and ventro-dorsal radiographic views of affected rabbits' abdomens, the stomach was moderately dilated and contained heterogeneous ingesta surrounded with a gas pattern, and large pockets of gas were visible in the small intestine and cecum (Figure 1).

Autopsies were performed as soon as the affected rabbits died. Autopsy findings included gastric dilation and perforation, GI track filled with gas, a mass of food and hair in the stomach, greenish skin and fatty yellow liver (Figure 2 ). The time interval from the onset of diarrhea until death varied from 1 to 14 days. These results confirmed that stress increased in all the rabbits as a result of surgery, causing GI disorders in several severely stressed rabbits after surgery for allogeneic tracheal transplantation. Rabbits with GI disturbance had higher cortisol levels than the otherwise healthy rabbits (Table I, $p<0.05$ ).

Pain at the surgical site, drug injection, and reduced appetite were considered to contribute to elevated levels of stress in rabbits after surgery. We believe that rabbits should not be subjected to surgery that can lead to long-term stress or even death and consequently the inefficient use of experimental animals.

When GI disorders occur after surgery for biomedical research in rabbits, factors such as malnutrition, body imbalance, and reduction in the number of experimental animals will affect the results of research. Therefore, treatments including intestinal motility agents such as cisapride, fluid therapy, syringe feeding, or antibiotics can be administered to rabbits with GI disorder in order to relieve signs and symptoms $(2,9)$. In addition, it is important to choose the proper type and dose of analgesics in order to relieve postoperative pain. An adequately increased dose of tramadol was found to reduce stress levels (10). The problem in the present study was caused by painful surgery rather than a genetic abnormality (Figure 3). Above all, prevention of GI disorder is most important for biomedical research in rabbits. In this regard, rabbits are not considered to be an appropriate model for stressful surgeries.

\section{Acknowledgements}

This research was supported by Basic Science Research Program through the National Research Foundation of Korea (NRF) funded by the Ministry of Education (2015R1D1A1A01060583) and was supported by the Industrial Technology Innovation Program (No. 10048358) funded by the Ministry Of Trade, Industry \& Energy (MI, Korea).

\section{References}

1 Mapara M, Thomas BS and Bhat KM: Rabbit as an animal model for experimental research. J Dent Res 9: 111-118, 2012.

2 Molly V: Textbook of Rabbit Medicine, Second Edition. Amsterdam, Elsevier Health Sciences, pp. 311-313, 2014.

3 Patrick JM, Daniel HR and Christian E: Biology of the Laboratory Rabbit, Second Edition. San Diego, Academic Press, p. $339,1994$.

4 Anna M and Brigitte L: BSAVA Manual of Rabbit Medicine (British Small Animal Veterinary Association), First Edition. Singapore, John Wiley \& Sons, Inc., p. 177, 2014.

5 John EH, Patricia VT, Susan VW and Colette LW: Harkness and Wagner's Biology and Medicine of Rabbits and Rodents, Fifth Edition. Iowa, Wiley-Blackwell, pp. 305-306, 2010.

6 Mark AS, Karla AS and Ronald PW: The laboratory rabbit, guinea pig, hamster, and other rodents. First Edition, San Diego, Academic Press, pp. 517-518, 2012.

7 Campbell-Ward M: Gastrointestinal physiology and nutrition. In: Ferrets, Rabbits and Rodents: Clinical Medicine and Surgery. Quesenberry KE and Carpenter JW (eds.). St. Louis, ElsevierSaunders, pp. 183-192, 2012.

8 Huynh M, Vilmouth S, Gonzalez MS, Calvo Carrasco D, Di Girolamo N and Forbes NA: Retrospective cohort study of gastrointestinal stasis in pet rabbits. Vet Rec 175: 225-226, 2014.

9 Lichtenberger $\mathrm{M}$ and Lennox A: Updates and advanced therapies for gastrointestinal stasis in rabbits. Vet Clin North Am Exot Anim Pract 13: 525-541, 2010.

10 Udegbunam RI, Onuba AC, Okorie-Kanu C, Udegbunam SO, Anyanwu MU and Ifeanyi OL: Effects of two doses of tramadol on pain and some biochemical parameters in rabbits postgastrotomy. Comp Clin Pathol 24: 783-790, 2015.

Received April 19, 2017

Revised May 4, 2017

Accepted May 9, 2017 